\title{
ANÁLISIS DE LA INCIDENCIA DE LA VIOLENCIA INTRAFAMILIAR EN LA PROBLEMÁTICA DEL ADOLESCENTE INFRACTOR EN LA CIUDAD DE GUAYAQUIL: REVISIÓN DE LAS MEDIDAS SOCIOEDUCATIVAS \\ ANALYSIS OF THE INCIDENCE OF DOMESTIC VIOLENCE IN THE PROBLEMS OF THE ADOLESCENT OFFENDER IN THE CITY OF GUAYAQUIL: REVIEW OF SOCIOEDUCATIONAL MEASURES
}

Mercedes Cecilia Coronel Gómez, Mgs. Magíster en Planificación, Acreditación y Evaluación de la Educación Superior. Especialista en Derecho civil, compañías, niñez y adolescencia y Abogada de los Tribunales y Juzgados (Ecuador). Docente Tiempo Completo de la Facultad de Derecho y Gobernabilidad de la Universidad ECOTEC, Ecuador. mecoronel@ecotec.edu.ec

\section{ARTÍCULO DE REFLEXIÓN}

Recibido: 19 de junio de 2020

Aceptado: 9 de septiembre de 2020

\section{RESUMEN}

El objetivo del presente trabajo es establecer la problemática del adolescente infractor en relación a la incidencia de la violencia intrafamiliar en el inadecuado comportamiento de los adolescentes en la ciudad de Guayaquil; para realizar el estudio de investigación se analizaron variables de datos informativos, tipos de violencia, rendimiento escolar, etc., todos enfocados a determinar si este problema afecta a los adolescentes. Por tanto la violencia, en cualquiera de sus formas o tipos, tiene sus consecuencias, y tratándose del adolescente afecta gravemente a su comportamiento y a su normal desenvolvimiento. La falta de valores, de principios, de comunicación, de cooperación, la pasividad, la desestructuración de la familia, figura como posibles causas afectando directamente al núcleo familiar. Los resultados obtenidos en esta investigación determinan que la violencia y sus manifestaciones inciden en el inadecuado comportamiento del adolescente, con lamentables consecuencias para su vida emocional y psicológica. También se realizó un análisis de las medidas socioeducativas y el efecto en la seguridad ciudadana en la ciudad de Guayaquil.

Palabras clave: Adolescente, conductas, delito, violencia. 


\section{ABSTRACT}

The objective of this work is to establish the problem of the offending adolescent in relation to the incidence of domestic violence in the inappropriate behavior of adolescents in the city of Guayaquil; To carry out the research study, informational data variables, types of violence, school performance, etc. were analyzed, all focused on determining if this problem affects adolescents. Therefore, violence, in any of its forms or types, has its consequences, and in the case of adolescents, it seriously affects their behavior and normal development. The lack of values, principles, communication, cooperation, passivity, and the breakdown of the family, figure as possible causes directly affecting the family nucleus. The results obtained in this investigation determine that violence and its manifestations do affect the adolescent's inappropriate behavior, with unfortunate consequences for their emotional and psychological life. An analysis of the measures was also carried out.

Keywords: Adolescent, conduct, crime, violence

\section{INTRODUCCIÓN}

En el trayecto de la vida del adolescente, una serie de factores determinan su desempeño; obstaculizando e influyendo negativamente en su normal desarrollo. La presente investigación lleva por título: La problemática del adolescente infractor en relación a las conductas delictivas de en la ciudad de Guayaquil, un tema que en diferentes formas y de diversas maneras está presente en muchos casos en el adolescente guayaquileño, en particular del adolescente infractor.

Se puede asegurar que la violencia intrafamiliar constituye una consecuencia de la situación socioeconómica de la familia; pues, en no pocas ocasiones, los conflictos en el seno de la familia tienen su origen en el factor económico y alcanzan diversos grados de manifestación por el nivel cultural de la pareja, siendo los niños, los adolescentes, quienes resultan como las primeras víctimas de ello, a veces, con manifiestas consecuencias en su desenvolvimiento estudiantil y en sus relaciones interpersonales. Sin embargo, poco o nada se hace a nivel de sociedad, olvidando su misión en la formación de las futuras generaciones.

El tema tratado, como se ha manifestado, no ha sido aún objeto de un estudio detenido, si bien toda la sociedad está consciente de su existencia; no obstante, se han emprendido acciones relacionadas con la concientización de los padres en su deber para con sus hijos. En este sentido están encaminados: el Código Orgánico de la Niñez y Adolescencia como un conjunto de normas de protección; el Juzgado de la Niñez y Adolescencia como organismo para aplicar la justicia. 
Pero se olvida ante todo de la formación de los padres, el conocimiento de sus deberes y obligaciones para con su pareja y para con sus hijos, y el conocimiento del adolescente en su desempeño psicológico, como base para proporcionarle el trato que se merece.

El tema ha sido tratado, en primer lugar, en su contenido teórico-científico a través de un marco teórico estructurado en base a la Violencia y el inadecuado comportamiento de los adolescentes y de su realidad en nuestro país.

\section{REVISIÓN TEÓRICA}

\section{Violencia}

El concepto violencia ha recibido diferentes definiciones, con notables divergencias según disciplinas, objetivos de investigación, entre otros. Es así como existen puntos de definición extensos o restringidos de violencia. La forma más restringida de definir la violencia, supone identificarla con actos de violencia física entre personas (Estramiana, 2016).

De esta manera se podría definir la violencia como el uso intencionado de la fuerza física en contra de un semejante con el propósito de herir, humillar, etc.". Violencia sería todo aquello que impide que la gente satisfaga sus necesidades fundamentales, alimentación, vestido, vivienda, y también dignidad (Estramiana, 2016).

De acuerdo a todo esto tenemos entonces que la violencia está presente cuando los seres humanos se ven influidos de tal manera que sus realizaciones afectivas, somáticas y mentales, que están por debajo de sus realizaciones potenciales de modo que, cuando el potencial es mayor que lo efectivo, y ello es evitable, existe violencia (Estramiana, 2016).

\section{Violencia intrafamiliar}

Corresponde aquella violencia cuyos protagonistas (agresores y víctimas), mantienen algún tipo de relación de parentesco (lazos de sangre- real o supuestos - o matrimonio). Algunos autores reducen su significado y la identifican con la violencia que tiene lugar en el seno del hogar, es decir, entre personas que conviven. "Otros lo amplían, para incluir a personas que no necesariamente son familiares ni conviven con la víctima; pero son sentidas por ésta como miembros de su intimidad; de ésta manera se incluye la violencia ejercida por novios, ex - novios y en general, ex - parejas". (Herrera, 2017).

Gran parte de las investigaciones de violencia intrafamiliar se centran al estudio de tres colectivos: mujeres, niños y ancianos; que son considerados los tipos más comunes de violencia de este tipo (Herrera, 2017). 
En casi todas las sociedades, hasta épocas muy recientes, no ha habido un rechazo explícito hacia las diferentes manifestaciones de violencia intrafamiliar. Así es, como se puede considerar que no eran actos condenables, salvo que se haga público algún caso especialmente dramático (Herrera, 2017).

Formaban parte de la esfera íntima, de aquel espacio que debía ser protegido del resto de la sociedad y en gran medida, continúa siendo así. Es de esta manera como ciertos movimientos, los feministas, por ejemplo, luchan no solo por actos rechazables y denunciables que toman centro en las relaciones familiares sino su consideración como un problema social (Lorenz, 2016).

La Organización Panamericana de la Salud ha definido a la violencia intrafamiliar como: toda acción u omisión cometida por algún miembro de la familia en relación de poder, sin importar el espacio físico donde ocurra, que perjudique el bienestar, la integridad física, psicológica o la libertad y el derecho al pleno desarrollo del otro/a miembro de la familia (Lorenz, 2016).

\section{Causas y consecuencias de la violencia intrafamiliar}

La violencia, su crecimiento en la actualidad y su invisible presencia se origina por la falta de valores, principios en la familia y sociedad (Herrera, 2017).

Las causas de la violencia intrafamiliar son:

- La falta de respeto, comunicación, comprensión entre las personas.

- El alcohol, actúa desinhibiendo al que lo consume, incrementando la impulsividad y liberando los impulsos agresivos, con lo que muchos comportamientos violentos están precedidos por el consumo de alcohol.

- Las drogas también favorecen las conductas delictivas y violentas por un proceso de destrucción ética y social de la personalidad y por la necesidad imperiosa de procurarse la droga de forma más o menos inmediata (Herrera, 2017).

- Falta de control del carácter, de los impulsos, generando violencia.

Entre las causas más relevantes de la violencia intrafamiliar están la disfunción de la familia, el distanciamiento de sus miembros y las mutaciones en su comportamiento o actitudes mentales, estos efectos pueden ser:

- Psicofísicos: Pueden generar cambios psíquicos o físicos; los cambios psíquicos se refieren a la baja autoestima, estrés, tensión, y los físicos se presentan con golpes, heridas, hematomas, fracturas, etc.

- Psicosociales: Producen exclusión y marginación del núcleo social. 
- En lo personal: - Inseguridad: La víctima es incompetente, insegura, inestable, indecisa. Temor: Sentimiento común por el ambiente de violencia que sufre. - Vergüenza: El silencio es notorio por la situación de violencia que atraviesa. - Culpa: Sentimiento que afecta directamente a la víctima, sintiéndose responsable de su situación. - Aislamiento: Imposibilita a la víctima de poder comunicar, produciendo distanciamiento con sus seres más cercanos, pues siente que sólo ella atraviesa este tipo de problemas. (Herrera, 2017).

- En la salud: - Depresión: Enfermedad de tristeza profunda, desproporcionada a la situación.

Las estadísticas señalan que cada año se desintegran cientos de familias a causa de la violencia intrafamiliar, los más vulnerables son las mujeres, niños y adolescentes, dicha violencia afecta principalmente a los adolescentes que están en proceso de formación, es importante mencionar que si no se mitiga este problema estos adolescentes generarán comportamientos inadecuados generando un adolescente infractor con las graves consecuencia que significa para la sociedad. La violencia intrafamiliar es la causa de efectos devastadores que ponen en peligro la estructura de la familia y la sociedad.

Los más preocupante en este contexto son los niños y adolescentes que se crían en la calle abandonados sin ninguna persona adulta a su cargo, donde son expuestos a diferentes peligros, a medida que pasa el tiempo implementan estrategias de sobre vivencia que entre ellas está, el robar para alimentarse e incluso llegan a matar a las personas que obstaculicen su trabajo, el tratar de vengarse de las personas que les hicieron daño y generar al adolescente infractor.

\section{Situación actual del adolescente infractor}

\section{El adolescente infractor}

Para Josué Carrillo, en su trabajo "Inimputabilidad de los menores una oportunidad para cometer delitos", define que "La palabra infracción deriva del latín: infractio, de infringere, significa; quebrantar, hecho prohibido bajo amenaza de pena. Llámese adolescente infractor, a aquella persona menor de edad que tiene una conducta que la sociedad rechaza, cuando ha violado las normas y preceptos legales vigentes" (Carrillo, 2017).

Hablar de adolescente infractor tiene un sentido preciso y apunta a una persona menor de dieciocho años que ha cometido una acción en contra de la Ley; dicho en otras palabras, es adolescente infractor, sólo quien ha violado los dispositivos jurídicos previamente definidos como delito o contravención, y se le haya atribuido o imputado dicha violación a través de un proceso judicial, manteniendo el respeto estricto sobre sus derechos, así como de las garantías procesales, para que finalmente sea declarado responsable (Carrillo, 2017). 


\section{La edad y la responsabilidad penal}

Tanto la legislación penal, como la especializada en adolescentes infractores, han determinado ciertos límites entre la minoría y la mayoría de edad a efectos penales, respecto de los sujetos autores de cualquiera de los comportamientos descritos en un tipo legal de delito (Carrillo, 2017).

En el Ecuador se entiende por "menores de edad" sujetos de responsabilidad penal especial por el cometimiento de un delito, a todas las personas comprendidas en los límites de edad mínimo y máximo legalmente consignados, esto es, quienes cumplen catorce años de edad hasta que alcanzan los dieciocho años (Carrillo, 2017).

Sin embargo, en el panorama regional y mundial, no existen criterios unánimes al momento de establecer, especialmente, la minoría de edad penal, por el contrario, se puede advertir discrepancias sobre los criterios normativos para la fijación de límites mínimos y máximos de edad penal.

El sistema penal ecuatoriano manifiesta que "un adolescente que comete un delito es inimputable tal como se desprende del CONA, donde se establece que los adolescentes son penalmente inimputables $y$, por tanto, no serán juzgados por jueces penales ordinarios ni se les aplicaran las sanciones previstas en las leyes penales" (Carrillo, 2017).

Es decir, no se les puede aplicar la ley penal cuando son responsables de un acto que en condiciones similares a una persona mayor de dieciocho años se le aplicarían las establecidas en el Código Orgánico Integral Penal, siendo acreedores simplemente a medidas socioeducativas, es decir, se colige que no le otorga la capacidad para responder por un hecho penal, pero el legislador, quien es el llamado a hacer los cambios legales en el país, debe considerar que la pena tiene que ser proporcional a la importancia social del hecho, es decir al impacto que produce en la sociedad, sin dejar de considerar que no puede tener responsabilidad quien no es sujeto de derechos y obligaciones (Carrillo, 2017).

\section{Sujetos intervinientes en procesos del adolescente infractor}

La Dirección Nacional de Policía Especializada para niños, niñas y adolescentes (Dinapen) es la institución encargada de velar por los derechos de los menores de edad del país que son encontrados en situación de riesgo

Juez de niñez y adolescencia

Fiscal de niñez y adolescencia

CAI - Centro de adolescentes infractores (masculino o femenino) 


\section{Unidad}

La Unidad de Adolescentes Infractores se encarga de la investigación de los jóvenes que tienen conflicto con la Ley Penal.

No todos los adolescentes pueden ser investigados por esta Unidad, solo aquellos cuyas acciones u omisiones contravienen normas establecidas en el Código Orgánico Integral Penal.

\section{Competencias de la Unidad.}

- En ningún caso un niño o una niña (entre 0 y 11 años, 11 meses y 31 días) pueden ser detenidos por la Policía Nacional.

- De ocurrir alguna conducta irregular, deben ser protegidos por la Dirección Nacional de Policía Especializada para Niños, Niñas y Adolescentes (DINAPEN), y entregados inmediatamente a sus padres, representantes legales y/o encargados de su custodia.

- $\quad$ La privación de libertad de un adolescente es resuelta en 24 horas por parte de un Juez Especializado en Adolescentes Infractores, según lo determina la Constitución de la República.

- Los procesos seguidos contra adolescentes en conflicto con la Ley Penal, son resueltos rápidamente (30 días), y no pueden durar más de 90 días en ningún caso, según lo establece el Código de la Niñez y Adolescencia.

- La Unidad de Adolescentes Infractores en Quito cuenta con cuatro fiscales especializados, y también aporta a la prevención de estas conductas con capacitación gratuita a entidades educativas que deseen conocer y prevenir las infracciones cometidas por los menores.

\section{Etapas}

1. La Instrucción Fiscal;

2. La Audiencia Preliminar;

3. La Audiencia de Juzgamiento; y,

4. La Etapa de Impugnación.

\section{Pasos de audiencia de Juzgamiento}

Inicia la secretaria de Juzgado

- Menciona el número de proceso

- La sustanciación de la causa 
- Presenta al Juez de niñez y adolescencia y a los sujetos procesales (fiscal especializado en niñez y adolescencia y defensor público y/o abogado particular y la víctima )

Fundamentos de hecho y de derecho

- Explicación de fundamentos de hecho

- Explicación de fundamentos de derecho

- Presentación de las pruebas

El juez dictará sentencia al finalizar la audiencia de juzgamiento

\section{Procedimiento Niñez y Adolescencia}

En la Ley de la Niñez y Adolescencia se regula así el procedimiento para sancionar a los adolescentes infractores:

- En el artículo 334: El ejercicio de la acción para el juzgamiento del adolescente corresponde únicamente al fiscal.

- En el artículo 335: Los sujetos procesales son los Fiscales de Adolescentes Infractores, el adolescente procesado y la víctima.

- En el artículo 340: El proceso para el juzgamiento del adolescente tiene las siguientes etapas:

1. Instrucción. (En caso de flagrancia hay una audiencia de flagrancia para que vaya a juicio)

2. Evaluación y Preparatoria de Juicio.

3. Juicio.

- En el artículo 342: El fiscal podrá investigar todos los hechos que lleguen a su conocimiento en donde se presuma la participación de un adolescente en el cometimiento de una infracción penal. La investigación previa no durará más de cuatro meses en los delitos de hasta 5 años de pena, y durará hasta ocho meses en los delitos con pena superior a cinco años. Si el fiscal obtiene elementos de convicción suficientes, solicitará al juzgador fije fecha y hora para la audiencia de formulación de cargos.

- En el artículo 342-A: Cuando se sorprenda a un adolescente en infracción flagrante se le aprehenderá y se realizará la audiencia de calificación de flagrancia y de legalidad para formular cargos y solicitar medidas cautelares.

- En el artículo 342-B: El adolescente que padezca un trastorno mental transitorio, no será responsable penalmente, aunque el juzgador le dictará medidas de seguridad.

- En el artículo 343: La etapa de instrucción durará cuarenta y cinco días improrrogables, contados a partir de la fecha de la audiencia de formulación de cargos. En caso de delito flagrante, la instrucción no excederá de treinta días. 
También es posible la vinculación en la instrucción de otro adolescente cuando se presuma su participación a través de una audiencia con una posibilidad de extender la duración por veinte días más por una sola vez.

- En el artículo 344: Cuando concluya el plazo de la instrucción fiscal, si existen elementos contundentes se solicitará al juzgador fecha y hora para la audiencia evaluatoria y preparatoria de juicio. En caso de no hallar elementos de convicción, el fiscal procederá a emitir un dictamen abstentivo para que le juzgador dicte su sobreseimiento.

- En el artículo 357: En el mismo anuncio de su decisión de convocar a audiencia de juzgamiento, el Juez fijará día y hora para su realización y ordenará el examen bio - sico - social del adolescente que deberá practicarse por la Oficina Técnica antes de la audiencia. Esta audiencia deberá llevarse a cabo dentro de un plazo no menor de diez ni mayor de quince días contados desde la fecha del anuncio.

\section{Sanciones - Medidas socio educativas para el adolescente infractor}

El Código de la Niñez y Adolescencia manifiesta:

Aplicación de las medidas. - La resolución que establezca la responsabilidad de un adolescente por un hecho tipificado como infracción penal, deberá imponerle una o más de las medidas socioeducativas, observando, en todos los casos, el principio de proporcionalidad contemplado, según la siguiente distinción.

1. Para los casos de contravenciones se aplicará obligatoriamente la medida de amonestación y una o más de las siguientes medidas:

a) Amonestación e imposición de reglas de conducta, de uno a tres meses,

b) Orientación y apoyo familiar; de uno a tres meses,

c) Servicios a la comunidad, de siete días a un mes; y

d) internamiento domiciliario, de siete días a tres meses (CONA, 2003)

2. Para los casos de infracciones que en la legislación penal ordinaria son sancionadas con prisión, se aplicara obligatoriamente la medida de amonestación y una o más de las siguientes medidas:

Amonestación e imposición de reglas de conducta de uno a seis meses;

Orientación y apoyo familiar, de tres a seis meses,

Servicios a la comunidad; de uno a seis meses,

d) Libertad asistida, de tres meses a un año;

e) Internamiento domiciliario, de tres meses a un año

f) Internamiento de fin de semana, de uno a seis meses 
g) Internamiento con régimen de semi libertad, de tres meses a dos años; (CONA, 2003)

Para los casos de infracciones que en la legislación penal ordinarias son sancionadas con reclusión, se aplicará obligatoriamente las medidas de amonestación y una o más de las siguientes medidas:

a) Libertad asistida hasta por 12 meses,

b) Internamiento con régimen de semi libertad hasta por 24 meses, y,

c) Internamiento institucional, hasta por cuatro años (CONA, 2003)

Los adolescentes, cuya medida de internamiento institucional exceda de 24 meses, tienen derecho a beneficiarse de la rebaja de tiempo por buen comportamiento, de modo que cada día del cual se pueda certificar su buen comportamiento y aprovechamiento en el estudio, en la capacitación laboral y en el trabajo, se cuente como dos.

Esta calificación deberá suscribirse por el Director y el Secretario del Equipo Técnico del centro de internamiento, y será remitida al juez cada mes".

En la proporcionalidad está la medida socio-educativa aplicada y la infracción atribuida al menor, la que tiene que ser considerada para determinar la sanción que tiene que recibir el infractor, siempre en beneficio a los intereses de los menores.

Cuando las infracciones que en la legislación penal ordinaria son sancionadas con reclusión, tiene que aplicarse con obligatoriedad la medida de amonestación y una o más de las establecidas en el Código de la Niñez y la Adolescencia, como: libertad asistida, internamiento con régimen de semi libertad y el internado institucional hasta por cuatro años, además tienen derecho a beneficiarse de la rebaja de tiempo por buen comportamiento.

Estas medidas son demasiadas contemplativas, ya que no fueron elaboradas pensando en la sociedad toda que es la perjudicada, sino solamente en un grupo de personas que con o sin razón delinquen por el simple de hecho de no sentirse a gusto con su realidad, por rebeldía o por el motivo que ellos crean necesario.

Con esto se ha conseguido que se llegue a incrementar la cantidad de actos delictivos a las normas penales por parte de los menores, ya que al saberse protegidos y con unas sanciones que son hasta placenteras, su comportamiento no va a mejorar peor a cambiar que es que se supone se pretende lograr con estas medidas socio-educativas.

\section{ANÁLISIS DE RESULTADOS}

La protección que necesitan los menores es necesaria, pero es también necesaria la protección de la sociedad, no se debe ni puede permitir que se cometan delitos como los asesinatos y homicidios por parte de los adolescentes, que entran y salen de los centros de reclusión con 
mucha facilidad, por lo cual se debería enmendar este error y sancionar como tiene que ser este delito con rigor, para precautelar la vida tanto del adolescente como de la sociedad en general.

Estar atentos a las sanciones que reciben los menores que han cometido infracciones a la ley penal y se ha demostrado su culpabilidad. Es necesario realizar reformas a la Normativa de niñez y adolescencia en relación a los menores comprendidos entre los 16 y 18 años de edad que cometan delitos de homicidio y asesinato, con el fin de que se los pueda sancionar de acuerdo al Código Orgánico Integral Penal.

\section{CONCLUSIONES}

Se concluye con el criterio doctrinal la necesidad de reformas legales para imputar y responsabilizar penalmente, a los menores comprendidos entre los 14 y 18 años de edad, porque nuestras leyes están facilitando la delincuencia juvenil.

Se concluye que sí hay implicancia entre inimputalidad y responsabilidades penales porque se relacionan. El inimputable no es responsable penal, mientras que el imputable si es responsable de las infracciones penales.

Tanto la doctrina como el más alto porcentaje de investigación concluyen en la necesidad de realizar un análisis jurídico de la legislación ecuatoriana que trata de la responsabilidad del adolescente infractor para combatir la delincuencia juvenil y la proliferación de bandas y pandillas juveniles que azotan y perturban la convivencia social de las grandes urbes.

En relación a lo investigado se concluye que la legislación ecuatoriana debe analizar específicamente el Código de la Niñez y Adolescencia porque allí se libra de responsabilidad a los menores infractores.

Se establece que los menores comprendidos entre los 14 y 18 años de edad, están suficientemente desarrollados en todos los aspectos, por lo que están en capacidad de discernir entre el bien y el mal para identificar lo que es delito.

La protección que necesitan los menores es necesaria, pero es también necesaria la protección de la sociedad, no se debe ni puede permitir que se cometan delitos como los asesinatos y homicidios por parte de los adolescentes, que entran y salen de los centros de reclusión con mucha facilidad, por lo cual se debería enmendar este error y sancionar como tiene que ser este delito con rigor, para precautelar la vida tanto del adolescente como de la sociedad en general.

Estar atentos a las sanciones que reciben los menores que han cometido infracciones a la ley penal y se ha demostrado su culpabilidad. Es necesario realizar reformas en relación a los 
menores comprendidos entre los 16 y 18 años de edad que cometan delitos de homicidio y asesinato, con el fin de que se los pueda sancionar de acuerdo al Código Orgánico Integral Penal.

\section{REFERENCIAS BIBLIOGRÁFICAS}

Arias, F. (2012). El proyecto de Investigación: Introducción a la metodología científica (6ta. ed.). 2012: Editorial Episteme.

Baena, G. (2014). Metodología de la Investigación. México: Editorial Patria.

Cabanellas, G. (2014). Diccionario Jurídico Elemental. Buenos Aires- Argentina: Ediciones Sudamerica.

Carbonell, J. (2012). Una Educación para mañana. Madrid: Ed.LIMUSA.

Carrillo, J. (2017). Inimputabilidad de los menores una oportunidad para cometer delitos.

CONA. (2003). Código Orgánico de la Niñez y Adolescencia. Quito-Ecuador: CEP.

Constituyente, A. (2008). CONSTITUCIÓN DE LA REPÚBLICA DEL ECUADOR. QuitoEcuador: CEP.

Estado, F. G. (2019). Informe de Evolución De delitos (2019). Quito-Ecuador: FCG.

Estramiana, Á. (2016). Fundamentos sociales del comportamiento. Barcelona-España: Ed.UOC.

Hernández Sampieri, R. (2016). Metodología de la investigación (Sexta ed.). (E. M. Hill, Ed.) México D.F., México, México: Editorial MC Graw Hill.

Herrera, J. (2017). Violencia Intrafamiliar: tipologías y causas de la Violencia Intrafamiliar. México: Editorial Leyer.

Kerlinger. (2017). Investigación del comportamiento. En Kerlinger, Investigación del comportamiento. Ed. Interamericana.

Lorenz. (2016). Biología del comportamiento: raíces instintivas de la agresión, el miedo y la libertad. Buenos Aires- Argentina: Ed.PP.

Miklebust, H. (1965). Trastornos del aprendizaje. Editorial Científico-Médica.

Nacional, A. (2014). Código Orgánico Integral Penal. Quito-Ecuador: Registro Oficial.

Nacional, A. (2018). Ley Orgánica de Educación Intercultural. Quito-Ecuador: CEP. Obtenido de https://educacion.gob.ec/ley-organica-de-educacion-intercultural-loei/

Piaget, J. (2017). Psicología del niño. Madrid: Ed.Morata.

Ramos, J. (2015). Manual sobre violencia familiar y sexual. México DF: Ed.LIMUSA.

Robbins, S. (2015). Comportamiento Organizacional. México: Ed. LIMUSA.

Sampieri, R. (2014). Metodoloía de la investigación. En R. Sampieri, Metodoloía de la investigación (pág. 45). MC Graw. 
Schiamberg, L. (1983). Funciones de la familia. Ediciones ABC.

Tamayo, C., \& Silva, I. (2014). Técnicas e instrumentos de recolección de datos. ChimbotePerú: Ediciones Lyris.

Trabajo, O. I. (2003). Declaración Universal de los Derechos Humanos. OIT.

UNESCO. (2018). Estrategia de la UNESCO sobre la educación para la salud y el bienestar. 